Central Washington University

ScholarWorks@CWU

All Faculty Scholarship for the College of the Sciences

College of the Sciences

$7-22-2020$

Contact in the Unitary Fermi Gas across the Superfluid Phase Transition

S. Jensen

Christopher N. Gilbreth

Y. Alhassid

Follow this and additional works at: https://digitalcommons.cwu.edu/cotsfac

Part of the Quantum Physics Commons 


\title{
Contact in the Unitary Fermi Gas across the Superfluid Phase Transition
}

\author{
S. Jensen $\odot,{ }^{1}$ C. N. Gilbreth $\oplus^{2, *}$ and Y. Alhassid $\oplus^{1}$ \\ ${ }^{1}$ Center for Theoretical Physics, Sloane Physics Laboratory, Yale University, New Haven, Connecticut 06520, USA \\ ${ }^{2}$ Department of Physics, Central Washington University, Ellensburg, Washington 98926, USA
}

(Received 9 July 2019; accepted 19 June 2020; published 22 July 2020)

\begin{abstract}
A quantity known as the contact is a fundamental thermodynamic property of quantum many-body systems with short-range interactions. Determination of the temperature dependence of the contact for the unitary Fermi gas of infinite scattering length has been a major challenge, with different calculations yielding qualitatively different results. Here we use finite-temperature auxiliary-field quantum Monte Carlo (AFMC) methods on the lattice within the canonical ensemble to calculate the temperature dependence of the contact for the homogeneous spin-balanced unitary Fermi gas. We extrapolate to the continuum limit for 40, 66, and 114 particles, eliminating systematic errors due to finite-range effects. We observe a dramatic decrease in the contact as the superfluid critical temperature is approached from below, followed by a gradual weak decrease as the temperature increases in the normal phase. Our theoretical results are in excellent agreement with the most recent precision ultracold atomic gas experiments. We also present results for the energy as a function of temperature in the continuum limit.
\end{abstract}

DOI: 10.1103/PhysRevLett.125.043402

Introduction.-The unitary Fermi gas (UFG) describes a system of spin- $1 / 2$ particles with a short-range interaction and a diverging $s$-wave scattering length $a$, which saturates the upper bound on the modulus of the scattering amplitude imposed by the unitarity condition. The UFG is of interest for understanding the properties of other systems such as high $-T_{c}$ superconductors $[1,2]$ and nuclear matter [3,4], and has been realized experimentally with ${ }^{6} \mathrm{Li}$ and ${ }^{40} \mathrm{~K}$ ultracold atomic Fermi gases [5-7]. Its quantitative understanding presents a challenge to theorists and experimentalists.

The contact $C$ is of fundamental importance in quantum many-body systems with short-range interactions. It describes the short-range correlations of particles of opposite spin and is defined by

$$
\int d^{3} R g_{\uparrow, \downarrow}^{(2)}(\mathbf{R}+\mathbf{r} / 2, \mathbf{R}-\mathbf{r} / 2) \underset{r \rightarrow 0}{\sim} \frac{C}{(4 \pi r)^{2}},
$$

where $g_{\uparrow, \downarrow}^{(2)}\left(\mathbf{r}_{\uparrow}, \mathbf{r}_{\downarrow}\right)=\left\langle\hat{n}_{\uparrow}\left(\mathbf{r}_{\uparrow}\right) \hat{n}_{\downarrow}\left(\mathbf{r}_{\downarrow}\right)\right\rangle$ is the pair correlation function, and $\hat{n}_{s_{z}}(\mathbf{r})$ the density of particles at position $\mathbf{r}$ with spin projection $s_{z}=\uparrow, \downarrow$. Several exact relations involving the contact, known as Tan's relations, were derived in Refs. [8-10]. In particular, the contact characterizes the high-momentum tail of the normalized momentum distribution $n_{s_{z}}(\mathbf{k})$ through the relation $n_{s_{z}}(\mathbf{k}) \underset{k \rightarrow \infty}{\sim} C / k^{4}$, where $k$ is the wave number and the distribution is normalized with $N_{s_{z}}=\int\left[d^{3} k /(2 \pi)^{3}\right] n_{s_{z}}(\mathbf{k}) \quad\left(N_{s_{z}}\right.$ being the total number of particles with spin projection $s_{z}$ ) [8]. The contact also characterizes the high-frequency tail of the shear viscosity spectral function [11,12]. It can be expressed in terms of the adiabatic derivative (at constant entropy $S$ ) of the thermal energy $E$ with respect to the inverse scattering length [9]

$$
C=\left.\frac{4 \pi m}{\hbar^{2}} \frac{\partial E}{\partial(-1 / a)}\right|_{S} .
$$

Other relations involving the contact were introduced in Refs. [13-28]; see Ref. [29] for a review.

Tan's relations were verified experimentally in the ultracold atomic gas experiments of Refs. [30,31]. Subsequently, the temperature dependence of the contact for the UFG was measured in a trap [32], and for the homogeneous system [33]. Reference [33] observed a sharp decrease in the contact as the temperature is lowered below the superfluid critical temperature $T_{c}$. Recently, two independent precision experiments $[34,35]$ measured the temperature dependence of the contact across the superfluid phase transition. The experiments agree well with each other and show a dramatic increase in the contact as the temperature is lowered below $T_{c}$.

Calculating the temperature dependence of the contact for the UFG has proved challenging, and published results differ widely $[12,12,36-40]$. This is not surprising given that many of the theoretical results were derived using uncontrolled approximations. However, two recent works $[39,40]$ rely on methods that have, in principle, controlled errors. Reference [39] used a diagrammatic Monte Carlo approach on a lattice [41] both in the superfluid and normal phases. Reference [40] used the bold diagrammatic Monte Carlo method [42] in the normal phase only.

Here we use an improved finite-temperature auxiliaryfield quantum Monte Carlo (AFMC) [43] method on a 
spatial lattice to calculate the temperature dependence of the contact across the superfluid transition for 40,66, and 114 particles. Our AFMC method works in the canonical ensemble and uses an algorithm we recently introduced [44-46] that enables calculations for much larger lattices than would otherwise be feasible. For each of these particle numbers, we extrapolate to the continuum limit, eliminating systematic errors due to a finite filling factor (or equivalently finite effective range $r_{e}$ [47]).

Our calculations of the contact are in remarkable agreement with the recent precision experiments of Refs. [34,35] both below and above $T_{c}$. Compared with available theoretical results for the contact, our calculations provide the best quantitative agreement with these experimental results across the superfluid phase transition. The temperature dependence we find is qualitatively similar to that found in the diagrammatic Monte Carlo approach [39] at temperatures below the critical temperature $T \lesssim T_{c} \simeq 0.15 T_{F}$ (where $T_{F}$ is the Fermi temperature), but exhibits a different behavior above $T_{c}$. The behavior we find for $T>T_{c}$ is similar to that of the bold diagrammatic Monte Carlo method [40], but is systematically lower.

We also calculate the temperature dependence of the thermal energy in the continuum limit for 40 and 66 particles, and compare it with the experimental results of Ref. [48]. Taking the zero-temperature limit of the thermal energy, we estimate the Bertsch parameter (measuring the ground-state energy in units of the free Fermi gas energy) to be $\xi=0.367(7)$, in agreement with the experimental value $\xi=0.376(5)$.

Lattice formulation. - We discretize space with a cubic lattice of linear size $L=N_{L} \delta x$, where $\delta x$ is the lattice spacing. We use periodic boundary conditions and take a zero-range interaction of strength $V_{0}$, i.e., $V=V_{0} \delta\left(\mathbf{r}-\mathbf{r}^{\prime}\right)$. The corresponding lattice Hamiltonian is

$$
\hat{H}=\sum_{\mathbf{k}, s_{z}} \epsilon_{\mathbf{k}} \hat{a}_{\mathbf{k}, s_{z}}^{\dagger} \hat{a}_{\mathbf{k}, s_{z}}+g \sum_{\mathbf{x}} \hat{n}_{\mathbf{x}, \uparrow} \hat{n}_{\mathbf{x}, \downarrow},
$$

where $g=V_{0} /(\delta x)^{3}$ is the coupling constant determined by

$$
\frac{1}{V_{0}}=\frac{m}{4 \pi \hbar^{2} a}-\int_{B} \frac{d^{3} k}{(2 \pi)^{3} 2 \epsilon_{\mathbf{k}}}
$$

to produce the given scattering length $a$ on the lattice ( $a \rightarrow \infty$ for the UFG). The integral over the wave vector $\mathbf{k}$ is restricted to the first Brillouin zone $B$ of the reciprocal lattice in momentum space of a spatial cubic lattice $\mathbf{x}=\left(n_{x}, n_{y}, n_{z}\right) \delta x, \quad n_{i} \in\{-M,-M+1, \ldots, M\}, \quad$ where $M=\left(N_{L}-1\right) / 2$ (we use odd $\left.N_{L}\right)$. The operators $\hat{a}_{\mathbf{k}, s_{z}}^{\dagger}$ and $\hat{a}_{\mathbf{k}, s_{z}}$ are, respectively, the creation and annihilation operators of a particle with wave vector $\mathbf{k}$ and spin projection $s_{z}=\uparrow, \downarrow$ obeying fermionic anticommutation relations $\left\{\hat{a}_{\mathbf{k}, s_{z}}^{\dagger}, \hat{a}_{\mathbf{k}^{\prime}, s_{z}^{\prime}}\right\}=\delta_{\mathbf{k}, \mathbf{k}^{\prime}} \delta_{s_{z}, s_{z}^{\prime}}$. The operator $\hat{n}_{\mathbf{x}, s_{z}}=$ $\hat{\psi}_{\mathbf{x}, s_{z}}^{\dagger} \hat{\psi}_{\mathbf{x}, s_{z}}$ is the number operator of particles at lattice site $\mathbf{x}$ with spin projection $s_{z}$, where $\hat{\psi}_{\mathbf{x}, s_{z}}^{\dagger}$ and $\hat{\psi}_{\mathbf{x}, s_{z}}$ are the creation and annihilation operators satisfying $\left\{\hat{\psi}_{\mathbf{x}, s_{z}}^{\dagger}, \hat{\psi}_{\mathbf{x}^{\prime}, s_{z}^{\prime}}\right\}=\delta_{\mathbf{x}, \mathbf{x}^{\prime}} \delta_{s_{z}, s_{z}}$. Here we use a quadratic single-particle dispersion relation $\epsilon_{\mathbf{k}}=\hbar^{2} \mathbf{k}^{2} / 2 m$. In the Supplemental Material [49] we show that dispersion relations used in other works $[39,41,54-56]$ lead to similar results after extrapolation to the continuum limit.

For a given lattice size $N_{L}^{3}$ and particle number $N$, there is a systematic error that arises from the finite lattice filling factor $\nu=N / N_{L}^{3}$, and an extrapolation $\nu \rightarrow 0$ is necessary to obtain the continuum limit for the given particle number. In the limit of low filling factor, the many-body energies scale as $\nu^{1 / 3}[41,47,57]$. We therefore use a linear fit in $\nu^{1 / 3}$ for our low-filling-factor simulations to extract the continuum results. The thermodynamic limit is then approached for large values of $N$.

Improved AFMC algorithm.-For the large lattice calculations, we employed an improved AFMC algorithm in which the calculation at finite temperature scales more gently than the usual $O\left(N_{s}^{3}\right)$, where $N_{s}=N_{L}^{3}$. At the temperatures of interest, and for a given auxiliary-field configuration, only a relatively small number $N_{\text {occ }}$ of significantly occupied single-particle states contribute to the observables; the remaining states can be omitted with a controllable error that can be made arbitrarily small. The calculation of the partition function in the occupied space then scales as $O\left(N_{\mathrm{occ}}^{2} N_{s}\right)$, though with an $O\left(N_{s}^{2}\right)$ calculation to determine $N_{\text {occ }}$. Typically we find that $N_{\text {occ }}$ is a small multiple $(\sim 2-4)$ of the number of particles $N$, so that the overall computational time for large lattices is reduced dramatically. This method was discussed and used in Refs. [44,45], and is described in detail in Ref. [46].

Results.-We performed AFMC simulations in the canonical ensemble as described in Ref. [45]. The simulations are carried out for $N=40,66$, and 114 particles, on lattices of size $N_{L}^{3}=5^{3}, 7^{3}, 9^{3}, 11^{3}, 13^{3}$, and $15^{3}$. We divide the inverse temperature $\beta=1 / k_{B} T$ ( $k_{B}$ is the Boltzmann constant), into discrete time slices of length $\Delta \beta$ (using the Trotter product for the propagator $e^{-\beta \hat{H}}$ ) and perform the simulations for several values of $\Delta \beta$. We then extrapolate to the limit $\Delta \beta \rightarrow 0$ using the quadratic $\Delta \beta$ dependence that characterizes the symmetric Trotter decomposition, thus removing the systematic error introduced by the finite $\Delta \beta$. Results for multiple lattice sizes $N_{L}^{3}$ for a given particle number $N$ are used to extrapolate to the continuum limit $\nu \rightarrow 0$ (see the Supplemental Material [49] for detailed extrapolation results). In the following we discuss results for two measurable thermal observables: the contact and the thermal energy.

(i) Contact: The expression (2) for the contact can also be written as

$$
C=\left.\frac{4 \pi m}{\hbar^{2}} \frac{\partial F}{\partial(-1 / a)}\right|_{T}
$$




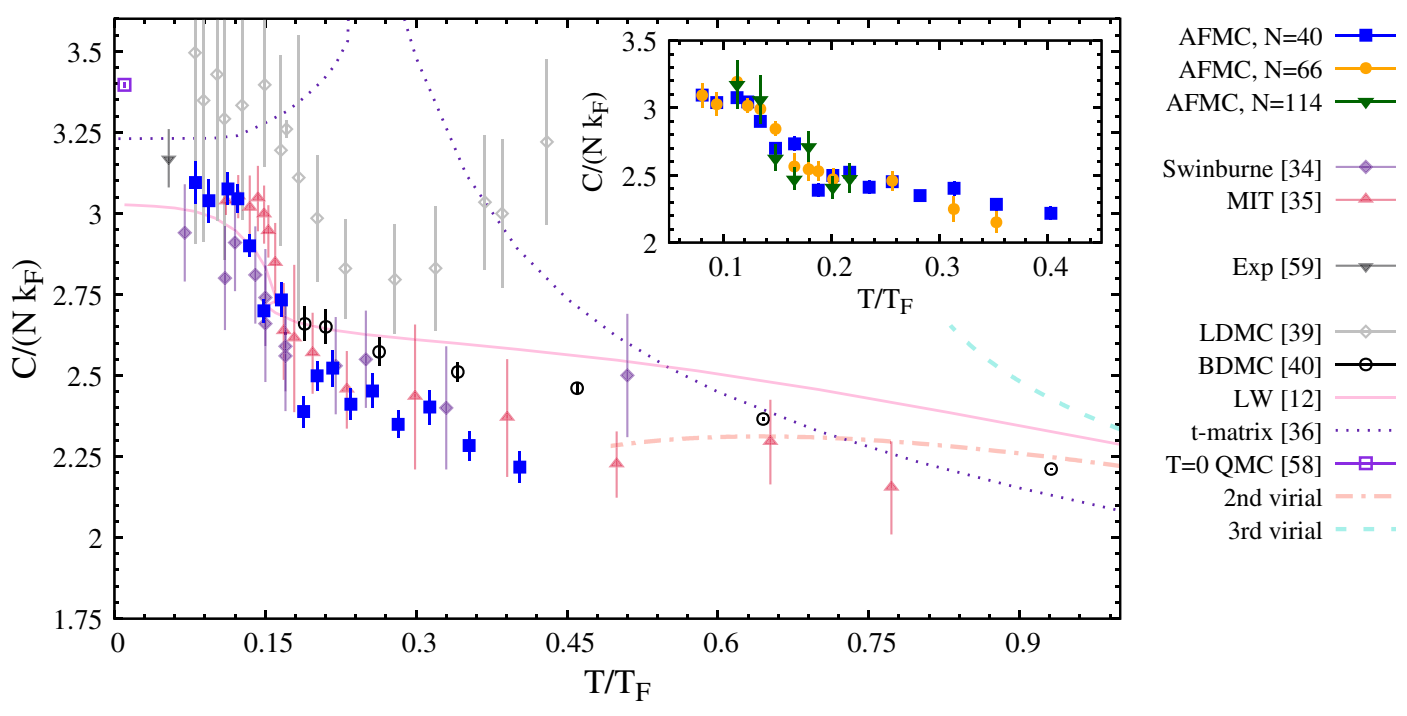

FIG. 1. The contact $C$ (in units of $N k_{F}$ ) of the UFG as a function of temperature $T$ (in units of $T_{F}$ ). Our AFMC results in the continuum limit for $N=40$ particles (solid blue squares) are compared with the recent experimental results of the Swinburne group [34] (solid purple diamonds) and the MIT group [35] (solid red up triangles). We also compare with other theoretical results: the lattice diagrammatic Monte Carlo (LDMC) results of Ref. [39] (open gray diamonds), the bold diagrammatic Monte Carlo (BDMC) results of Ref. [40] (open black circles), the Luttinger-Ward (LW) results of Ref. [12] (solid pink line), and the $t$-matrix result of Ref. [36] (dotted purple line). We also show the $T=0$ quantum Monte Carlo results of Ref. [58] (open purple square) and the low-temperature experimental result of Ref. [59] (solid black down triangle). The second-order and third-order virial expansions for the contact are shown, respectively, by the dashed-dotted red line and dashed blue line. Virial coefficients were calculated in Refs. [38,60-62]. The inset shows our continuum limit AFMC results for several particle numbers: $N=40$ (solid blue squares), $N=66$ (solid orange circles), and $N=114$ (solid green down triangles).

where $F$ is the free energy and the derivative is evaluated at constant temperature $T$. In the lattice formulation the contact can then be calculated from

$$
C=\frac{m^{2} V_{0}\langle\hat{V}\rangle}{\hbar^{4}},
$$

where $\langle\hat{V}\rangle$ is the thermal expectation value of the potential energy $\hat{V}=g \sum_{\mathbf{x}} \hat{n}_{\mathbf{x}, \uparrow} \hat{n}_{\mathbf{x}, \downarrow}$. In Fig. 1 we show our AFMC results for the temperature dependence of the contact $C$ calculated from Eq. (6) in the continuum limit in units of $N k_{F}$ for $N=40$ particles (solid blue squares). The temperature $T$ is expressed in units of the Fermi temperature $T_{F}=\varepsilon_{F} / k_{B}$, where $\varepsilon_{F}=\left(\hbar^{2} / 2 m\right)\left(3 \pi^{2} \rho\right)^{2 / 3}$ is the Fermi energy for a free gas of density $\rho=\nu /(\delta x)^{3}$. Our results are in excellent agreement with the recent experimental results of the Swinburne group [34] (solid purple diamonds) and of the MIT group [35] (solid red up triangles), both above and below the critical temperature $T_{c}$.

We also compare our results with the theoretical calculations of Refs. [12,36,38-40,58,60-62] and the lowtemperature experimental result of Ref. [59].

Our results for the contact show similar qualitative behavior to those of the lattice diagrammatic Monte Carlo method of Ref. [39] (open gray diamonds) in the low-temperature regime, but have markedly different qualitative behavior for $T>T_{c}$. Our results above $T_{c}$ are more consistent with the bold diagrammatic Monte Carlo results of Ref. [40] (open black circles), but are systematically lower.

In Fig. 1, we also compare our AFMC results for the contact with those of Ref. [12] (solid pink line), where good overall qualitative agreement is seen for the entire temperature range. This is somewhat surprising since the work of Ref. [12] used the Luttinger-Ward approach, which contains uncontrolled systematic errors. However, this method has been shown to produce reliable results for other observables of the UFG [45,63]. Quantitatively, our results are above those of Ref. [12] at low temperatures, and significantly below them for $T>T_{c}$.

Reference [37] used an AFMC approach similar to the current work but in the grand-canonical ensemble, and extracted the contact above $T_{c}$ from the tail of the momentum distribution at a finite filling factor. The calculated temperature dependence of the contact in Ref. [37] differs substantially from our results. As shown in Fig. 3 of the Supplemental Material [49], the contact is very sensitive to the filling factor, particularly at temperatures $T>T_{c}$, and the continuum extrapolation leads to qualitatively different results.

We tested our continuum extrapolations by comparing the results of different dispersion relations for the singleparticle energy. For a finite filling factor $\nu$ the contact depends on the dispersion relation, but similar results 


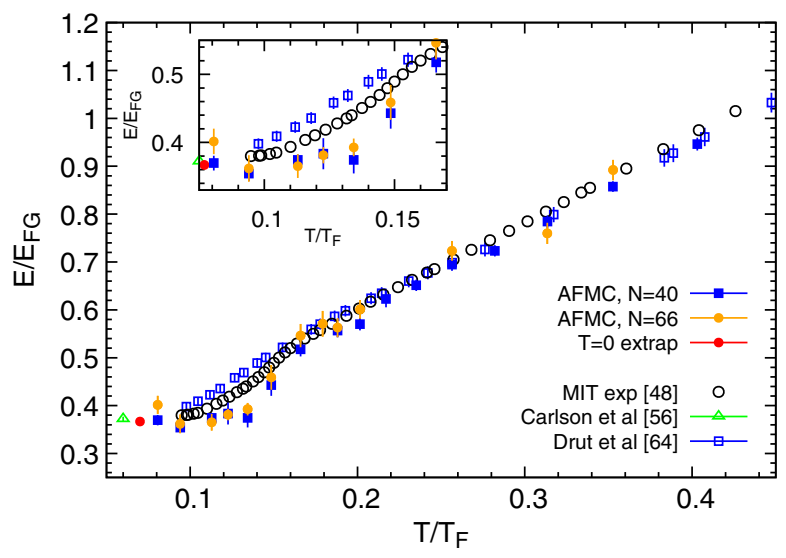

FIG. 2. AFMC thermal energy $E$ (in units of the Fermi gas ground-state energy $E_{\mathrm{FG}}$ ) as a function of temperature $T$ (in units of the Fermi temperature $T_{F}$ ) for the UFG obtained in the continuum limit for $N=40$ particles (solid blue squares) and $N=66$ particles (solid orange circles). We compare with the experimental results of Ref. [48] (open black circles), and with the AFMC results of Ref. [64] (open blue squares). Using our lowest temperature results, we estimate the Bertsch parameter to be $\xi=0.367(7)$ (solid red circle), in close agreement with the ground-state quantum Monte Carlo estimate $\xi=0.372(5)$ of Ref. [56] (open green triangle). The inset shows the low-temperature regime.

should be obtained in the limit $\nu \rightarrow 0$. In Fig. 5 of the Supplemental Material [49], we show the contact for multiple dispersion relations for $N=40$ particles at $T / T_{F} \simeq 0.24$ and demonstrate that they extrapolate to similar values (within statistical errors) in the continuum limit. In the comparison we use a quadratic dispersion (the one implemented in our calculations), the hopping dispersion $\epsilon_{\mathbf{k}}^{(h)}=\left(\hbar^{2} / m \delta x^{2}\right)\left[3-\sum_{i} \cos \left(k_{j} \delta x\right)\right]$ (used in Ref. [39]), and the dispersion $\epsilon_{k}^{(3)}=\left(\hbar^{2} k^{2} / 2 m\right)$ $\left[1-\alpha(k \delta x / \pi)^{2}\right]$ with $\alpha=0.257022$ [56].

The inset of Fig. 1 shows the continuum contact results for $N=40,66$, and 114 particles. The results for $N=66$ and 114 particles show little systematic difference from the $N=40$ particle results, although the latter have smaller statistical errors. This suggests that our results for the contact are close to the thermodynamic limit.

Our calculations are limited to $T \lesssim 0.45 T_{F}$. Large lattice simulations with lower filling factors are necessary to determine the contact at higher temperatures up to $T / T_{F} \approx 1$, where a meaningful comparison with the virial expansion results can be made.

(ii) Thermal energy: We also calculated the thermal energy $E=\langle\hat{H}\rangle$ of the UFG (in units of the zero-temperature free Fermi gas energy $\left.E_{\mathrm{FG}}=\frac{3}{5} N \varepsilon_{F}\right)$ as a function of temperature $T$ (measured in units of the Fermi temperature $\left.T_{F}\right)$. In Fig. 2 we show our AFMC results for $E / E_{\mathrm{FG}}$ as a function of $T / T_{F}$ in the continuum limit for $N=40$ (solid squares) and $N=66$ (solid circles) particles. We compare our results with the experimental results of Ref. [48] (open
TABLE I. Various estimates of the Bertsch parameter $\xi$.

\begin{tabular}{lcc}
\hline \hline Method & $\xi$ & Error \\
\hline Fixed-node diffusion Monte Carlo [66] & 0.42 & 0.01 \\
Duke experiment [67] & 0.39 & 0.02 \\
ENS experiment [68,69] & 0.41 & 0.01 \\
Ground-state fixed-node Monte Carlo [70] & $\leq 0.383$ & 0.001 \\
Ground-state AFMC [56] & 0.372 & 0.005 \\
MIT experiment [48] & 0.376 & 0.005 \\
Lattice quantum Monte Carlo [65] & 0.366 & +0.016 \\
AFMC (this work) & 0.367 & 0.007 \\
\hline \hline
\end{tabular}

circles), the AFMC results of Ref. [64] (open squares) and the zero-temperature quantum Monte Carlo result of Ref. [56] (open triangle).

In the high-temperature regime we find good quantitative agreement between our results and those of Refs. [48] and [64]. Below $T_{c} \simeq 0.15 T_{F}$, the AFMC results of Ref. [64] are systematically above our results. This is anticipated since the results of Ref. [64] were calculated at a finite filling factor of $\nu \simeq 0.040-0.045$ (corresponding to a nonnegligible effective range parameter $k_{F} r_{e} \simeq 0.36$ for the quadratic dispersion relation), while here we use a continuum extrapolation to remove the systematic error associated with a finite filling factor. Compared with the experimental results of Ref. [48], our results are systematically lower below $T_{c}$.

We can use our low-temperature results to extract the Bertsch parameter $\xi$ defined by $E(T=0)=\xi E_{\mathrm{FG}}$. Taking an average of its values for our lowest two temperatures and for both 40 and 66 particles, we find $\xi=0.367(7)$. In Table I we compare values of $\xi$ determined from recent experimental and theoretical works. Our result is in agreement with the value $\xi=0.372(5)$ found in the $N=66$ ground-state Monte Carlo calculation of Ref. [56], and with the lattice Monte Carlo result $\xi=0.366_{-0.011}^{+0.016}$ of Ref. [65]. Our value for $\xi$ also agrees with the experimental value $\xi=0.376(5)$ of Ref. [48].

Conclusions.-We carried out canonical-ensemble lattice AFMC simulations for the UFG for $N=40,66$, and 114 particles using an improved AFMC algorithm. Our results for each particle number include extrapolations to the continuum limit of zero filling factor $\nu \rightarrow 0$, thus eliminating systematic errors due to finite-range effects. In particular, we calculated the temperature dependence of the contact across the superfluid phase transition, and found excellent agreement with the recent experimental results of Refs. [34,35]. Compared with previous calculations of the temperature dependence of the contact, our AFMC results provide the best quantitative agreement with these recent experimental results.

It would be interesting to apply our methods to determine the continuum limits of other thermodynamic observables of the UFG and use them as benchmarks for various theoretical methods. 
We thank K. Van Houcke, N. Navon, and F. Werner for useful discussions. We also thank J. Carlson, J. E. Drut, T. Enss, O. Goulko, M. J. H. Ku, B. Mukherjee, P. Pieri, C. J. Vale, and M. W. Zwierlein for providing the data shown in Figs. 1 and 2. This work was supported in part by the U.S. DOE Grants No. DE-FG02-91ER40608, No. DESC0019521, and No. DE-FG02-00ER41132. The research presented here used resources of the National Energy Research Scientific Computing Center (NERSC), a U.S. Department of Energy Office of Science User Facility operated under Contract No. DE-AC02-05CH11231. We also thank the Yale Center for Research Computing for guidance and use of the research computing infrastructure.

*Present address: Honeywell Quantum Solutions, Broomfield, Colorado 80021, USA.

[1] M. Randeria, Nat. Phys. 6, 561 (2010).

[2] E. J. Mueller, Rep. Prog. Phys. 80, 104401 (2017).

[3] J. Carlson, S. Gandolfi, and A. Gezerlis, Prog. Theor. Exp. Phys. 2012, 01A209 (2012).

[4] S. Gandolfi, A. Gezerlis, and J. Carlson, Annu. Rev. Nucl. Part. Sci. 65, 303 (2015).

[5] C. A. Regal, M. Greiner, S. Giorgini, M. Holland, and D. S. Jin, Phys. Rev. Lett. 95, 250404 (2005).

[6] W. Ketterle and M. W. Zwierlein, Riv. Nuovo Cimento Soc. Ital. Fis. 31, 247 (2008).

[7] B. Mukherjee, Z. Yan, P. B. Patel, Z. Hadzibabic, T. Yefsah, J. Struck, and M.W. Zwierlein, Phys. Rev. Lett. 118, 123401 (2017).

[8] S. Tan, Ann. Phys. (Amsterdam) 323, 2952 (2008).

[9] S. Tan, Ann. Phys. (Amsterdam) 323, 2971 (2008).

[10] S. Tan, Ann. Phys. (Amsterdam) 323, 2987 (2008).

[11] E. Taylor and M. Randeria, Phys. Rev. A 81, 053610 (2010).

[12] T. Enss, R. Haussmann, and W. Zwerger, Ann. Phys. (Amsterdam) 326, 770 (2011).

[13] R. Combescot, S. Giorgini, and S. Stringari, Europhys. Lett. 75, 695 (2006).

[14] M. Punk and W. Zwerger, Phys. Rev. Lett. 99, 170404 (2007).

[15] G. Baym, C. J. Pethick, Z. Yu, and M. W. Zwierlein, Phys. Rev. Lett. 99, 190407 (2007).

[16] E. Braaten and L. Platter, Phys. Rev. Lett. 100, 205301 (2008).

[17] E. Braaten, D. Kang, and L. Platter, Phys. Rev. A 78, 053606 (2008).

[18] S. Zhang and A. J. Leggett, Phys. Rev. A 77, 033614 (2008).

[19] F. Werner, L. Tarruell, and Y. Castin, Eur. Phys. J. B 68, 401 (2009).

[20] S. Zhang and A. J. Leggett, Phys. Rev. A 79, 023601 (2009).

[21] P. Pieri, A. Perali, and G. C. Strinati, Nat. Phys. 5, 736 (2009).

[22] R. Combescot, F. Alzetto, and X. Leyronas, Phys. Rev. A 79, 053640 (2009).

[23] W. Schneider, V. B. Shenoy, and M. Randeria, arXiv:0903.3006.

[24] H. Hu, X.-J. Liu, and P. D. Drummond, Europhys. Lett. 91, 20005 (2010).
[25] E. Braaten, D. Kang, and L. Platter, Phys. Rev. Lett. 104, 223004 (2010).

[26] D. T. Son and E. G. Thompson, Phys. Rev. A 81, 063634 (2010).

[27] Y. Nishida, Phys. Rev. A 85, 053643 (2012).

[28] J. Hofmann and W. Zwerger, Phys. Rev. X 7, 011022 (2017).

[29] E. Braaten, in The BCS-BEC Crossover and the Unitary Fermi Gas (Springer, New York, 2012), pp. 193-231.

[30] J. T. Stewart, J. P. Gaebler, T. E. Drake, and D. S. Jin, Phys. Rev. Lett. 104, 235301 (2010).

[31] E. D. Kuhnle, H. Hu, X.-J. Liu, P. Dyke, M. Mark, P. D. Drummond, P. Hannaford, and C. J. Vale, Phys. Rev. Lett. 105, 070402 (2010).

[32] E. D. Kuhnle, S. Hoinka, P. Dyke, H. Hu, P. Hannaford, and C. J. Vale, Phys. Rev. Lett. 106, 170402 (2011).

[33] Y. Sagi, T. E. Drake, R. Paudel, and D. S. Jin, Phys. Rev. Lett. 109, 220402 (2012).

[34] C. Carcy, S. Hoinka, M. G. Lingham, P. Dyke, C. C. N. Kuhn, H. Hu, and C. J. Vale, Phys. Rev. Lett. 122, 203401 (2019).

[35] B. Mukherjee, P. B. Patel, Z. Yan, R. J. Fletcher, J. Struck, and M. W. Zwierlein, Phys. Rev. Lett. 122, 203402 (2019).

[36] F. Palestini, A. Perali, P. Pieri, and G. C. Strinati, Phys. Rev. A 82, 021605(R) (2010).

[37] J. E. Drut, T. A. Lähde, and T. Ten, Phys. Rev. Lett. 106, 205302 (2011).

[38] H. Hu, X.-J. Liu, and P. D. Drummond, New J. Phys. 13, 035007 (2011).

[39] O. Goulko and M. Wingate, Phys. Rev. A 93, 053604 (2016).

[40] R. Rossi, T. Ohgoe, E. Kozik, N. Prokof'ev, B. Svistunov, K. Van Houcke, and F. Werner, Phys. Rev. Lett. 121, 130406 (2018).

[41] E. Burovski, N. Prokof'ev, B. Svistunov, and M. Troyer, New J. Phys. 8, 153 (2006).

[42] K. Van Houcke, F. Werner, T. Ohgoe, N. V. Prokof'ev, and B. V. Svistunov, Phys. Rev. B 99, 035140 (2019).

[43] For a recent review, see Y. Alhassid, Auxiliary-field quantum monte carlo methods in nuclei, in Emergent Phenomena in Atomic Nuclei from Large-Scale Modeling: A Symmetry-Guided Perspective, edited by K. D. Launey (World Scientific, Singapore, 2017), pp. 267-298.

[44] S. Jensen, C. N. Gilbreth, and Y. Alhassid, Eur. Phys. J. Special Topics 227, 2241 (2019).

[45] S. Jensen, C. N. Gilbreth, and Y. Alhassid, Phys. Rev. Lett. 124, 090604 (2020).

[46] C. N. Gilbreth, S. Jensen, and Y. Alhassid, arXiv:1907 .10596.

[47] F. Werner and Y. Castin, Phys. Rev. A 86, 013626 (2012).

[48] M. J. H. Ku, A. T. Sommer, L. W. Cheuk, and M. W. Zwierlein, Science 335, 563 (2012).

[49] See Supplemental Material at http://link.aps.org/ supplemental/10.1103/PhysRevLett.125.043402 for additional details and for the data used to generate the figures. The Supplemental Material includes Refs. [50-53].

[50] C. N. Gilbreth and Y. Alhassid, Phys. Rev. A 88, 063643 (2013).

[51] C. N. Gilbreth and Y. Alhassid, Comput. Phys. Commun. 188, 1 (2015). 
[52] A. Bulgac, J. E. Drut, and P. Magierski, Phys. Rev. Lett. 96, 090404 (2006).

[53] A. Bulgac, J. E. Drut, and P. Magierski, Phys. Rev. A 78, 023625 (2008).

[54] E. Burovski, N. Prokof'ev, B. Svistunov, and M. Troyer, Phys. Rev. Lett. 96, 160402 (2006).

[55] O. Goulko and M. Wingate, Phys. Rev. A 82, 053621 (2010).

[56] J. Carlson, S. Gandolfi, K. E. Schmidt, and S. Zhang, Phys. Rev. A 84, 061602(R) (2011).

[57] L. Pricoupenko and Y. Castin, J. Phys. A 40, 12863 (2007).

[58] S. Gandolfi, K. E. Schmidt, and J. Carlson, Phys. Rev. A 83, 041601(R) (2011).

[59] S. Hoinka, M. Lingham, K. Fenech, H. Hu, C. J. Vale, J. E. Drut, and S. Gandolfi, Phys. Rev. Lett. 110, 055305 (2013).

[60] X. Leyronas, Phys. Rev. A 84, 053633 (2011).

[61] X.-J. Liu, Phys. Rep. 524, 37 (2013).

[62] M. Sun and X. Leyronas, Phys. Rev. A 92, 053611 (2015).
[63] W. Zwerger, Strongly interacting Fermi gases, in Proceedings of the International School of Physics "Enrico Fermi"-Course 191 "Quantum Matter at Ultralow Temperatures”, edited by M. Inguscio, W. Ketterle, S. Stringari, and G. Roati (IOS Press, Amsterdam, SIF, Bologna, 2016), pp. 63-141.

[64] J. E. Drut, T. A. Lähde, G. Wlazłowski, and P. Magierski, Phys. Rev. A 85, 051601(R) (2012).

[65] M. G. Endres, D. B. Kaplan, J.-W. Lee, and A. N. Nicholson, Phys. Rev. A 87, 023615 (2013)

[66] G. E. Astrakharchik, J. Boronat, J. Casulleras, and S. Giorgini, Phys. Rev. Lett. 93, 200404 (2004).

[67] L. Luo and J. E. Thomas, J. Low Temp. Phys. 154, 1 (2009).

[68] S. Nascimbène, N. Navon, K. Jiang, F. Chevy, and C. Salomon, Nature (London) 463, 1057 (2010).

[69] N. Navon, S. Nascimbène, F. Chevy, and C. Salomon, Science 328, 729 (2010).

[70] M. M. Forbes, S. Gandolfi, and A. Gezerlis, Phys. Rev. Lett. 106, 235303 (2011). 